\title{
Nitric Oxide Production in the Striatum and Cerebellum of a Rat Model of Preterm Global Perinatal Asphyxia
}

\author{
M. Barkhuizen ${ }^{1,2,3,4}$ • W. D. J. Van de Berg ${ }^{1,2,5}$ • J. De Vente $^{2}$ • C. E. Blanco ${ }^{1}$ • \\ A. W. D. Gavilanes ${ }^{1,3,6}$ • H. W. M. Steinbusch ${ }^{2,3,7}$
}

Received: 13 September 2016 / Revised: 30 December 2016 / Accepted: 2 January 2017 / Published online: 21 January 2017

(C) The Author(s) 2017. This article is published with open access at Springerlink.com

\begin{abstract}
Encephalopathy due to perinatal asphyxia (PA) is a major cause of neonatal morbidity and mortality in the period around birth. Preterm infants are especially at risk for cognitive, attention and motor impairments. Therapy for this subgroup is limited to supportive care, and new targets are thus urgently needed. Post-asphyxic excitotoxicity is partially mediated by excessive nitric oxide (NO) release. The aims of this study were to determine the timing and distribution of nitric oxide (NO) production after global PA in brain areas involved in motor regulation and coordination. This study focused on the rat striatum and cerebellum, as these areas also affect cognition or attention, in addition to their central role in motor control. $\mathrm{NO}$ /peroxynitrite levels were determined empirically with a fluorescent marker on postnatal days P5, P8 and P12. The distributions of neuronal NO synthase (nNOS), cyclic guanosine monophosphate (cGMP), astroglia and caspase- 3 were determined with immunohis-
\end{abstract}

M. Barkhuizen and W. D. J. Van de Berg contributed equally to this work

Electronic supplementary material The online version of this article (doi:10.1007/s12640-017-9700-6) contains supplementary material, which is available to authorized users.

H. W. M. Steinbusch

h.steinbusch@maastrichtuniversity.nl

1 Department Pediatrics, School for Mental Health and Neuroscience (MHeNs), Maastricht University, Maastricht, The Netherlands

2 Department Psychiatry and Neuropsychology, School for Mental Health and Neuroscience (MHeNs), Maastricht University, Maastricht, The Netherlands

3 EURON - European Graduate School of Neuroscience, Maastricht, The Netherlands tochemistry. Apoptosis was additionally assessed by measuring caspase-3-like activity from P2-P15. On P5 and P8, increased intensity of $\mathrm{NO}$-associated fluorescence and cGMP immunoreactivity after PA was apparent in the striatum, but not in the cerebellum. No changes in nNOS immunoreactivity or astrocytes were observed. Modest changes in caspase-3-activity were observed between groups, but the overall time course of apoptosis over the first 11 days of life was similar between PA and controls. Altogether, these data suggest that PA increases $\mathrm{NO} /$ peroxynitrite levels during the first week after birth within the striatum, but not within the cerebellum, without marked astrogliosis. Therapeutic benefits of interventions that reduce endogenous NO production would likely be greater during this time frame.

Keywords Asphyxia $\cdot$ Nitrosidative stress $\cdot$ cGMP $\cdot$ Neuronal nitric oxide synthase $\cdot$ Peroxynitrite $\cdot$ Selective vulnerability
4 DST/NWU Preclinical Drug Development Platform, North-West University, Potchefstroom, South Africa

5 Department of Anatomy and Neurosciences, Neuroscience Campus Amsterdam, VU University Medical Centre, Amsterdam, Netherlands

6 Institute of Biomedicine, Faculty of Medicine, Catholic University of Guayaquil, Guayaquil, Ecuador

7 Department of Translational Neuroscience, Faculty of Health, Medicine and Life Sciences, Maastricht University, P.O. Box 5800, 6212 AZ Maastricht, The Netherlands 


\section{Introduction}

Neonatal encephalopathy (NE) due to perinatal asphyxia (PA) is a common cause of morbidity and mortality in the period around birth. In 2010, 8.5 infants per 1000 live term-births developed PA-related encephalopathy (Lee et al. 2013). The long-term neurological outcome after NE varies, from normal neurocognitive functioning after mild asphyxia to cerebral palsy, epilepsy, cognitive, behavioural or memory problems in severe cases (Armstrong-Wells et al. 2010; Volpe 2012). PA-related injury to the preterm brain is even more complex, since prematurity by itself increases the risk of NE (Volpe 2009a). While preterm birth before 37 weeks of gestation occurs in 5-8\% of all pregnancies, very low gestational age (VLGA) birth, before 32 weeks of gestation occurs, in about $1 \%$ of singletons and 9\% of twin pregnancies (Schaaf et al. 2011). In cohorts of preterm infants with multifactorial encephalopathy, behavioural, cognitive, attention or social deficits have been reported in $25-50 \%$ of cases and 5-10\% had major motor impairments. A large portion of this disability burden was made up by VLGA infants, due to improved survival rates with modern medical care. Although the major motor impairments are striking, cognitive deficits are far more common (Volpe 2009a; Volpe 2009b). In preterm infants, PA causes both white matter injury of the developing oligodendrocytes (periventricular leukomalacia) in the sub-cortical regions and associated grey matter injury to the striatum and other basal ganglia structures, thalamus, basis pontis, brain stem and cerebellum (Cabaj et al. 2012; Logitharajah et al. 2009; Shah et al. 2006). Injury in these infants is a combination of primary destruction after PA and secondary maturational and trophic disease (Volpe 2009a).

PA occurs when oxygen supply between the mother and the foetus is disrupted, causing a biphasic brain injury. The acute injury results from the combined effects of cellular energy failure, acidosis, glutamate release, intracellular calcium accumulation, lipid peroxidation and nitric oxide (NO) neurotoxicity that disturb vital cell components. This results in cell death. From 6 to $48 \mathrm{~h}$ after the insult, a secondary cerebral energy failure occurs with mitochondrial dysfunction due to sustained pathological reactions in the primary phase (Perlman 2006; Piña-Crespo et al. 2014). The time-window for therapeutic intervention is thus limited. Currently, therapeutic hypothermia is the only therapy available for term infants with NE. This only partially reduces morbidity, and it is not used in preterm infants (Edwards et al. 2010). There is thus an urgent need for therapies in the preterm- and term infants. Animal models are essential tools for the preclinical development and testing of new therapies. In humans and large animals, the majority of brain development occurs prenatally. However, in rodents, the brain is still immature at birth and only resembles the term human infant at roughly 7-10 days postnatal (Semple et al. 2013). This also makes the rat a suitable organism to study insults to the preterm brain. Our group, and others, have optimized a rat model of global anoxia during birth to investigate PA in the rodent equivalent of the 23-32-week-old human foetus (Semple et al. 2013).

In this study, we focused on two structures important for motor control and coordination, which are in differential developing states at the time of insult, namely the striatum and the cerebellum. The primary roles of the striatum involve learning of associations between stimuli, actions and rewards (Balleine et al. 2007), the selection between competing response alternatives and motivational modulation of motor behaviour (Lenz and Lobo 2013; Liljeholm and O'Doherty 2012). In humans, neurogenesis of the striatal medium spiny neurons begins around week 11.5 and striatal synaptogenesis begins around 13 weeks of gestation. Synaptogenesis is well-established before mid-gestation, with near-uniform synapses by 34 weeks of human gestation (Freeman et al. 1995; Sarnat et al. 2013). The cerebellum undergoes rapid expansion from week 24 to 40 of gestation. During this time, the cerebellar volume increases by $3.5-5$-fold. The cerebellum is particularly vulnerable in preterm infants, due to its rapid growth towards the end of gestation (Volpe 2009b). The cortico-striato-cerebellar tract is instrumental for motor sequence learning (Tzvi et al. 2014). In addition to its established role in motor coordination, the cerebellum also directs linguistic and related cognitive and behavioral-affective functions (De Smet et al. 2013). Damage to these two regions is of clinical significance for both the motor deficits and cognitive/ attention deficits after PA (Volpe 2009b; Volpe 2012).

The NO cascade has emerged as both a major player in neurotoxicity - and a potential therapeutic intervention in PA. During PA, NO is involved in both the early phase injury and during secondary energy failure. Immediately after, asphyxia at birth neuronal nitric oxide synthase (nNOS) is activated, increasing NO neurotransmission. After 12-24 h, inducible nitric oxide synthase (iNOS) is activated in glial cells which leads to cerebral NO production (Gunes et al. 2007; Perlman 2006). Concurrent increases in the generation of superoxide cause the formation of peroxynitrite $\left(\mathrm{ONOO}^{-}\right)$. Peroxynitrite is a potent oxidative agent which causes tissue injury and contributes to ischemic injury in the immature brain, by irreversibly inhibiting the mitochondrial respiratory chain (Ikeno et al. 2000; Weis et al. 2011). The newborn brain is especially vulnerable to this type of insult due to its relatively low antioxidant levels (McQuillen and Ferriero 2004; Shim and Kim 2013). The extent of excessive NO production is dependent on the severity/duration of asphyxia, with variability across different neuron types and nervous system locations (Dorfman et al. 2009; Klawitter et al. 2007). Nitrotyrosine - a product of peroxynitrite and proteins - was present in the brain tissue of human NE infants at autopsy (Groenendaal et al. 2006). Moreover, increased NO in the cerebrospinal fluid in the first $24 \mathrm{~h}$ correlates with the severity of the NE (Ergenekon et al. 2004; Gunes et al. 2007). The 
exact role of $\mathrm{NO}$ and peroxynitrite in mediating the effects of PA remains to be elucidated. Pharmacological inhibition of neuronal nitric oxide synthase (nNOS) has been proposed as a strategy to reduce cerebral palsy and other motor deficits after PA (Ji et al. 2009; Yu et al. 2011) and nNOS inhibitor potent neuroprotective agents in most animal models for hypoxia-ischemia and excitotoxicity in vitro. Selective inhibition of nNOS shows beneficial effects including preservation of mitochondrial function after in utero ischemia. nNOS inhibition also improves the short-term survival of GABAergic interneurons in the striatum in hypoxic ischemic preterm sheep and delays the onset of post-asphyxial seizures (Drury et al. 2014; Rao et al. 2011). Inhaled NO has shown beneficial effects in rodent models of neonatal stroke. However, high doses of NO, or low doses of NO, administered during the reperfusion period are detrimental (Charriaut-Marlangue et al. 2012)

The aim of this study was to determine the timing and regional selectivity of increased $\mathrm{NO}$ production global PA in brain areas as striatum and cerebellum, involved in motor regulation and coordination structures. Targeting supraphysiological NO production could be a fertile therapeutic target for the preterm infant. In order to use NO production for a therapeutic target, we need to know at which time points after PA NO production peaks, whether these peaks coincide with increased cell death and whether regional differences exist in the brain.

\section{Materials and Methods}

\section{Animals}

Full-term pregnant Wistar rats (Charles River-Broekmans, Someren, The Netherlands) and their male pups $(n=108)$ were used for the present study. Female pups were sacrificed at birth. The pups were divided into two groups (PA and control). Six pups per group were used at each time-point (suppl. Fig. S1). They were housed under standard conditions (12:12 h light: dark cycles, $\left.20^{\circ} \mathrm{C}\right)$ with free access to standard laboratory chow and water. The local Committee on Animal Welfare approved all animal care and procedures. Within this study, exclusively male offspring was used because both morphological and behavioural studies provided evidence for a differential vulnerability to a birth insult in males versus females. A greater impact is seen in the male sex, probably due to the protecting role of the circulating hormones in females (El-Khodor and Boksa 2003; Zhu et al. 2006).

\section{Induction of PA}

PA was induced in rat pups at $\mathrm{P} 0$ by placing the uteri and its contents in a water bath for $20 \mathrm{~min}$, as described previously
(Vlassaks et al. 2014). Briefly, time-pregnant Wistar dams were decapitated immediately after delivery of two pups (control vaginal deliveries) and rapidly hysterectomized. The uterus horns containing the remaining pups were placed in a water bath at $37^{\circ} \mathrm{C}$ for $20 \mathrm{~min}$ (severe PA). Afterwards, pups were removed from the uterus horns and stimulated to breath by cleaning their skin and by gently padding them on the chest. The pups were left to recover for $60 \mathrm{~min}$ in a paediatric incubator at $36.5^{\circ} \mathrm{C}$ and randomly assigned to a surrogate mother (10 pups per mother), which had given birth on the same day. The percentage of mortality in the PA and control group was, respectively, \pm 50 and $0 \%$.

\section{4,5-Diaminofluorescein Diacetate (DAF-2/DA) Detection in Slices}

For the DAF2/DA detection in fresh tissue, six rat pups were decapitated and their brains were rapidly removed and placed into ice-cold Krebs-Ringer bicarbonate buffer ( $\mathrm{pH} 7.4)$ aerated with $95 \% \mathrm{O}_{2} / 5 \% \mathrm{CO}_{2}$. The forebrain and the cerebellum were chopped into $300-\mu \mathrm{m}$ coronal slices using a McIllwain tissue chopper. Slices were separated under a microscope and transferred to a multi-well culture plate containing Krebs buffer $\left(4^{\circ} \mathrm{C} ; \mathrm{pH} 7.4\right)$ and $1 \mathrm{mM}$ isobutyl-methyl xanthine (IBMX) to inhibit 3,5'-cyclic nucleotide phosphodiesterase (PDE) activity. Alternated slices were transferred to a second multi-well plate and used for nNOS and cGMP immunohistochemistry. All slices were incubated in Krebs-Ringer buffer containing IBMX for $30 \mathrm{~min}$ and slowly warmed to 35.5 or $25.5^{\circ} \mathrm{C}$ under an atmosphere of $5 \% \mathrm{CO}_{2} / 95 \% \mathrm{O}_{2}$ prior to DAF2/DA staining.

DAF-2-triazole (DAF-2/T) fluorescence was studied in slices $(300 \mu \mathrm{m})$ including the striatum of rat pups at postnatal day P5, P8 and P12 ( $n=6$ per group at each time-point) using the DAF2/DA detection assay (Sigma, The Netherlands) according to the method of Lopez-Figueroa et al. (2000). Upon entry into the cell, DAF-2/DA is hydrolysed by cytosolic esterases, producing DAF-2. DAF-2 reacts with NO and peroxynitrite to form the highly fluorescent derivative DAF2/T (Bryan and Grisham 2007; Rodriguez et al. 2005; Roychowdhury et al. 2002). The slices were incubated with $1.5 \mathrm{ml} 10 \mu \mathrm{M}$ DAF-2/DA incubation buffer (150 mM Tris$\mathrm{HCl}, 3 \mu \mathrm{M}$ tetrahydrobiopterin, $1 \mu \mathrm{M}$ flavin adenine dinucleotide (FAD, Sigma), $1 \mu \mathrm{M}$ flavin mononucleotide (FMN, Sigma), $1 \mathrm{mM}$ NADPH, $0.6 \mathrm{mM} \mathrm{CaCl}_{2}$ (Merck) and $100 \mu \mathrm{M}$ L-arginine (Sigma) per well for $45 \mathrm{~min}$ at 35.5 or $25.5^{\circ} \mathrm{C}$. During incubation with the DAF-2/DA solution, lights were turned off and a dark box was placed over the incubation chamber. Following incubation with DAF-2/DA, the slices were washed in phosphate-buffered saline ( $\mathrm{pH}$ 7.4) and placed on non-coated slides and cover slipped using PBSglycerol (1:4). To confirm that DAF-2/DA has a high affinity for $\mathrm{NO}$, slices were incubated in the presence or absence of the 
NO donor $0.1 \mathrm{mM}$ sodium nitroprusside (SNP; Sigma) for $10 \mathrm{~min}$, or the NOS inhibitor $0.1 \mathrm{mM} N^{G}$-nitro-L-arginine (Sigma) for $30 \mathrm{~min}$. As a negative control, slices were incubated in media lacking DAF-2/DA.

\section{nNOS, cGMP and Caspase-3 Immunohistochemistry}

Slices of the striatum and cerebellum $(n=6,10-\mu \mathrm{m}$ thick sections adjacent to the slices used in the DAF-2/DA experiment) were used to visualize cGMP-producing and NOS active structures, using antisera against cGMP (de Vente et al. 1987) and nNOS (Herbison et al. 1996). After incubation in the presence or absence of SNP or $N^{G}$-nitro-L-arginine, slices were fixed for $2 \mathrm{~h}$ with $4 \%$ paraformaldehyde and cut into sections with a cryostat. The sheep anti-cGMP and anti-nNOS antisera were used, respectively, at a dilution of 1:4000 and 1:2000 and visualized using an Alexa-conjugated donkey anti-sheep antibody (1:100; Mol. Probes, USA). The nNOS and cGMP sections were processed to study co-localization with cell-specific markers. An antibody against glial fibrillary acidic protein (anti-GFAP) (1:1600; Sigma) was used to identify astrocytes $(n=5)$. The immunolabelling was visualized with a Cy3-conjugated donkey anti-mouse antiserum (1:800) or goat anti-mouse Alexa Fluor ${ }^{\circledR} 488$ (1:100; Mol. Probes, USA). The sections were incubated overnight at $4^{\circ} \mathrm{C}$ with the primary antibody followed by $2 \mathrm{~h}$ at room temperature with the secondary antibody. Negative controls were performed by omitting the primary antibody. nNOS immunoreactivity labelled low-threshold spiking interneurons in the striatum and the cerebellar granule cells. Parvalbumin immunohistochemistry identified the fast-spiking striatal interneurons and cerebellar Purkinje cells. Basket and stellate interneurons in cerebellar molecular layer express both parvalbumin and nNOS (Contestabile 2012; Lenz and Lobo 2013; Schwaller et al. 2002). Parvalbumin-immunoreactivity $(n=6)$ was visualized with a rabbit antiserum (1:1500) provided by P.C. Emson (Babraham Institute, Cambridge, UK) in combination with a donkey anti-rabbit biotinylated antiserum (1:400) and streptavidine-Cy3 (1:2000). Sections were cover slipped using TBS: glycerol (1:3). Sections were examined at a magnification of $\times 400$ and $\times 1000$ with an Olympus AXE-70 microscope.

For caspase-3 immunohistochemistry, another set of six pups were anaesthetized with sodium pentobarbital and perfused transcardially with a fixative containing $4 \%$ paraformaldehyde and $2 \%$ picric acid. Afterwards, the brains were snapfrozen and cut into $16-\mu \mathrm{m}$ thick coronal sections. These sections were incubated with a rabbit polyclonal anti-caspase-3 antibody (67341A; Pharmingen, Europe) diluted 1:500. The anti-caspase-3 antibody was visualized using a biotinylated goat anti-rabbit antibody (1:400; Jackson Immunoresearch Laboratories) and streptavidine Cy-3. Caspase-3 positive cells were counted in tissue of six rats of each group (PA and control) at P8 as described previously in Van de Berg et al. (2002). The total amount of caspase-3-positive cells was estimated by multiplying the number of counted cells in all sections by the sampling interval (i.e., equal to eight).

\section{Fluorometric Assay of Caspase-3-like Activity}

Another set of six control and six asphyctic rat pups was used for analyses of caspase-3-like activity at P2, P5 or P8, P11 and P15 in cerebellum homogenates. The cerebellum was collected and homogenized in a lysis buffer containing $137 \mathrm{mM}$ $\mathrm{NaCl}, 20 \mathrm{mM}$ Tris- $\mathrm{HCl}$ (pH 8.0), 1\% NP-40, 10\% glycerol and a complete protease inhibitor tablet (Roche, NL). The tissue samples were briefly centrifuged and an aliquot of the supernatant ( 30 or $50 \mu \mathrm{l}$ ) was used. The assay is based on fluorometric determination of the cleavage of the Ac-AspGlu-Val-Asp-AMC peptide (Ac-DEVD-AMC; Biomol, Germany) by caspase-3 as described in detail previously (Van den Hove et al. 2006). The cleavage was followed at 2 min intervals for $3 \mathrm{~h}$.

\section{Image Analysis}

Fluorescence intensity of the images obtained from the slices loaded with DAF-2/DA and immunostained sections were analysed using a macro designed for measuring grey values in a given area (region of interest, ROI). Background values were measured using the slices incubated with the incubation buffer lacking the DAF-2/DA or lacking the primary antibody. Measurements were performed on three sampled images of three systematically sampled slices. From these data, the mean grey value in the area was calculated. Digital images were captured using a CoolView CCD camera system attached to a MR C600 confocal microscopy (Leica Microsystems, Germany). The microscope was equipped with a narrowband MNIBA-type FITC filter, or MNG filter for CY fluorescence (Chroma Technology, The Netherlands). Excitation was measured at $488 \mathrm{~nm}$ and emission at $530 \mathrm{~nm}$. Grey scaled images were directly converted into artificial colours with the analySIS $®$ image analysis system. All sections were stained simultaneously and recorded on the same day to minimize experimental variation.

\section{Statistics}

Group comparison (controls vs. PA) of the number and caspase- 3 positive cells as well as the grey values of immunofluorescence intensity measurements were analysed using the Student $t$ test. The differences in Ac-DEVD-AMC cleavage between control and asphyctic rats were evaluated using a pairwise two-way ANOVA analysis and post hoc tests using Bonferroni correction for repeated measures. Statistics were carried out using the SigmaStat ${ }^{\mathrm{TM}}$ software version 2.03. 
Differences were considered significant if $P \leq 0.05$. All data are presented as mean \pm standard error of the mean (SEM).

\section{Results}

\section{Timing of Regional NO Production in the Rat Asphyctic Striatum and Cerebellum}

A bright fluorescent signal could be observed in the striatum and cerebellum of all pups after loading with DAF-2/DA. The fluorescent signal was localized intracellular in neuron-like cells and their proximal dendrites and in addition in vascular structures. Fluorescence in DAF-2 loaded slices of control rat pups was clearly visible throughout the entire striatum, predominantly in the dorso-medial part. Within the cerebellum, DAF-2/T fluorescence was visible throughout the molecular and granular cell layer (Fig. 1). The cerebellum had higher $\mathrm{NO}$ /peroxynitrite levels than the striatum at all time-points after birth. In control rat pups, the intensity of the DAF-2/T fluorescent signal in the striatum was higher at P5 and P8 than that at P12 (see Table 1). In the PA group, the intensity of the fluorescent signal was greater than in the control group at P5 and P8, but not at P12 (see Table 1). There was no apparent difference in fluorescence pattern or their intensity in the cerebellum between control and PA rats at P5, P8 or P12 (Fig. 1). The fluorescent signal was however less intense at P12 than at P5 or P8.

Pre-incubation with the NO donor SNP led to a major increase in the intensity and density of the DAF-2/T fluorescent signal in both striatum and cerebellum. Background levels were more intense after pre-incubation with SNP, and almost all cells showed an extremely bright diffuse fluorescence (see Fig. 2 and Table 1). Pre-incubation with $N^{G}$-L-nitro-arginine
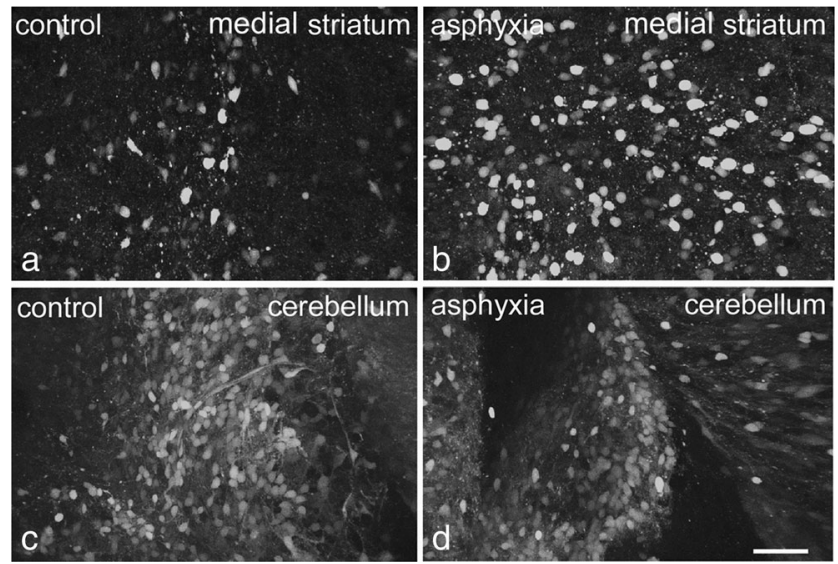

Fig. 1 DAF-2 fluorescence in tissue slices from medial striatum $(\mathbf{a}, \mathbf{b})$ and cerebellum $(\mathbf{c}, \mathbf{d})$ of a control $(\mathbf{a}, \mathbf{c})$ and asphyctic rat $(\mathbf{b}, \mathbf{d})$ at postnatal day 8 . Images were taken throughout the slice $(300 \mu \mathrm{m})$ with a confocal laser scanning microscope and combined into one image per area using an image analysis system. Scale bar is $50 \mu \mathrm{m}$ for all photographs
(0.1 mM, a NOS inhibitor) slightly suppressed the DAF-2/T fluorescent signal at P5, P8 and P12 in both striatum and cerebellum of $\mathrm{PA}$ rats at $35.5^{\circ} \mathrm{C}$. Lowering the incubation temperature to $25.5^{\circ} \mathrm{C}$ drastically lowered the signal.

Data on NO-mediated cGMP synthesis were obtained from slices incubated with $1 \mathrm{mM}$ IBMX to inhibit PDE activity. Incubation of the rat slices at P5, P8 and P12 with $1 \mathrm{mM}$ IBMX resulted in intense cGMP immunoreactivity (cGMPIR) in cell bodies and fibres in the striatum and cerebellum. Pre-incubation with $0.1 \mathrm{mM}$ NG-L-nitro-arginine abolished the cGMP signal, indicating that this NOS inhibitor abolished NOS activity, and as a consequence NO production and cGMP synthesis. Incubation of the slices with SNP $(0.1 \mathrm{mM}$, a NO donor) resulted in an increase of cGMP-IR in cells and fibres in both the striatum and cerebellum of both groups. The PA group had increased cGMP-IR in cells and fibres throughout the striatum compared to the control group at P5 and P8 $(P<0.05)$, but not at P12 $(P>0.05)$. nNOS-IR was observed in cell bodies and varicose fibres in grey and white matter of the striatum and cerebellum. Co-localization between cGMP and parvalbumin, but not between nNOS and parvalbumin, was found in cells and fibres in the striatum and cerebellum at P5, P8 and P12 (see Fig. 3). There was no detectable difference in nNOS-IR between control and asphyctic pups in the striatum or cerebellum. The results of the present study show that PA markedly increases $\mathrm{NO}$ /peroxynitrite during first postnatal week (P5 and P8, but not at P12), in the rat striatum. NO production in the cerebellum showed a similar trend, but no significant differences were observed between control and PA groups.

\section{Caspase-3 Distribution and Activity and Astrogliosis in the Rat Asphyctic Stratum and Cerebellum}

Caspase- 3 positive cell profiles were visible in both the grey and white matter of striatum and cerebellum of control and asphyctic pups during the first week of postnatal life. In the cerebellum, caspase-3-positive cells were found within white matter and in the granular cell layer at P2 and P5. At P8, caspase-3 positive cells were mainly observed within the granular cell layer. The caspase-3 immunohistochemistry was similar between the control and PA groups.

To assess the time course of caspase-3-like activity during normal development and after PA, we measured DEVD cleavage in homogenates of cerebellum of control and PA rats during the first 15 days of postnatal life. The results of DEVD cleavage activity are shown in Fig. 4. The pattern of DEVD cleavage in the cerebellum was stable during the first week of life, followed by a decrease at P11 (P8 vs. P11; $P<0.01$ ) and an increase at P15. The time course of DEVD cleavage activity was not different between control and asphyctic group and cerebellum, but the DEVD cleavage was moderately higher in the PA group at P8 and P11 (PA vs. control; $P<0.05$ ) and 
Table 1 Summary of the experiments performed with rat striatal slices at postnatal day $\mathrm{P} 5$, P8 and P12. Numbers represent the number of experiments. Per experiment, at least three slices throughout the striatum $(300 \mu \mathrm{m})$ were studied. For image scoring references, see Fig. 3 (absence in $3 \mathrm{E}$, weak in $3 \mathrm{C}, \mathrm{D}, \mathrm{F}$ strong in $3 \mathrm{~A}$, B, very strong in $3 \mathrm{G}, \mathrm{H}$ )

\begin{tabular}{|c|c|c|c|c|c|c|c|}
\hline \multirow[t]{2}{*}{ Age } & & \multicolumn{3}{|c|}{ Control } & \multicolumn{3}{|c|}{ Asphyxia } \\
\hline & & Basal & $\mathrm{N}^{\mathrm{G}}$-L-Nitro-arginine & SNP & Basal & $\mathrm{N}^{\mathrm{G}}$-L-Nitro-arginine & SNP \\
\hline \multirow[t]{4}{*}{ P5 } & Absence DAF & 0 & 1 & 0 & 0 & 2 & 0 \\
\hline & Weak DAF & 4 & 4 & 0 & 0 & 4 & 0 \\
\hline & Strong DAF & 4 & 0 & 5 & 6 & 1 & 3 \\
\hline & Very strong DAF & 0 & 0 & 2 & 2 & 0 & 4 \\
\hline \multirow[t]{4}{*}{ P8 } & Absence DAF & 0 & 2 & 0 & 0 & 1 & 0 \\
\hline & Weak DAF & 4 & 4 & 1 & 1 & 3 & 0 \\
\hline & Strong DAF & 3 & 0 & 3 & 4 & 3 & 6 \\
\hline & Very strong DAF & 0 & 0 & 2 & 3 & 0 & 1 \\
\hline \multirow[t]{4}{*}{ P12 } & Absence DAF & 1 & 3 & 0 & 0 & 5 & 0 \\
\hline & Weak DAF & 5 & 2 & 0 & 6 & 1 & 0 \\
\hline & Strong DAF & 0 & 0 & 6 & 1 & 0 & 1 \\
\hline & Very strong DAF & 0 & 0 & 0 & 0 & 0 & 5 \\
\hline
\end{tabular}
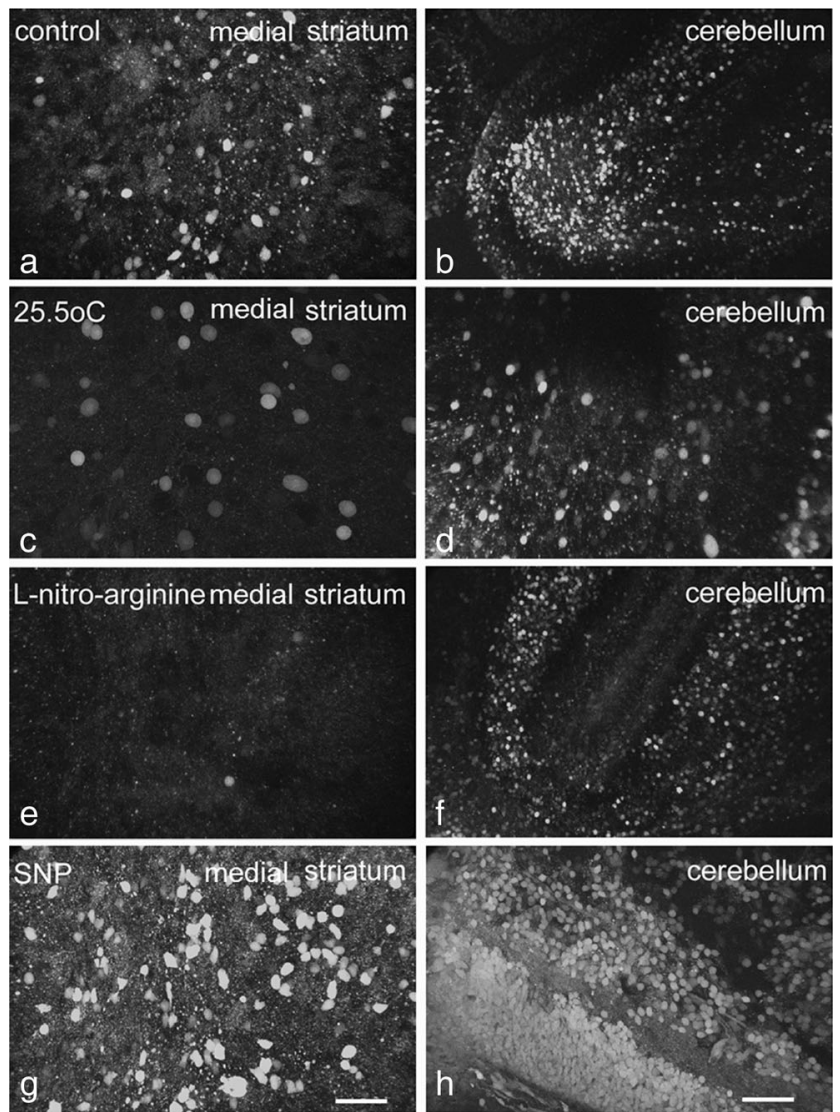

Fig. 2 DAF-2 fluorescence in tissue slices of the medial striatum (a) and cerebellum (b) of a control rat incubated in the presence of $1 \mathrm{mM}$ IBMX at $35.5^{\circ} \mathrm{C}$ for $45 \mathrm{~min}$ and $\mathbf{c}, \mathbf{d}$ in the presence of $1 \mathrm{mM} \mathrm{IBMX}$ at $25.5^{\circ} \mathrm{C}$ for $45 \mathrm{~min}$; $\mathbf{e}, \mathbf{f}$ incubated in the presence of $0.1 \mathrm{mM} N^{G}$-nitro-L-arginine at $35.5^{\circ} \mathrm{C}$ for $45 \mathrm{~min}$; and $\mathbf{g}$, $\mathbf{h}$ incubated in the presence of $0.1 \mathrm{mM} \mathrm{SNP}$ at $35.5^{\circ} \mathrm{C}$ for $45 \mathrm{~min}$. Hypothermia or incubation with $N^{G}$-nitro-Larginine led to a decrease in fluorescent signal in both the striatum $(\mathbf{c}, \mathbf{e})$ and cerebellum $(\mathbf{d}, \mathbf{f})$. Pre-incubation with SNP led to a strong increase of the fluorescent signal in both the striatum (g) and cerebellum (h). Scale bar is $50 \mu \mathrm{m}$ for all photographs lower at P15 (not significant). This corresponds to the cerebellar DAF-2/T fluorescence which also did not show large variation between groups and which was higher at $\mathrm{P} 5$ and $\mathrm{P} 8$ than that at later time-points. Overall, the regional distribution and timing of apoptosis in the striatum and cerebellum were similar between the PA and control groups in the first week, although there were modest relative increases in caspase- 3 cleavage in the PA group up until P11. Astrogliosis also did not vary significantly between the PA and control groups (Fig. 5).

\section{Discussion}

\section{Regional Differences in NO Production and Apoptosis}

In the striatum, we observed a supraphysiological increase in $\mathrm{NO}$ /peroxynitrite and cGMP, which was not present in the cerebellum. The striatal NO production was not accompanied by an increase in nNOS expression. This is consistent with observations that iNOS, rather than nNOS or eNOS, is upregulated after asphyxia. iNOS greatly increases NO concentrations, which leads to peroxynitrite formation (Ikeno et al. 2000). Another group showed decreased striatal nNOSpositive cells in organotypic cell cultures made 3 days after $\mathrm{PA}$, accompanied by PA-related increases in nNOSexpressing cells in the substantia nigra, a region that normally has lower nNOS activity than the striatum (Klawitter et al. 2006; Klawitter et al. 2007). In the cerebellum, the coupling between increased neuronal activity and local blood flow relies almost exclusively on NO (Rancillac et al. 2006). Thus, under physiological conditions, the cerebellum has the highest NOS activity and the highest concentration of glutamate and aspartate in the brain (Blanco et al. 2010). NO/peroxynitrite production in the striatum and cerebellum was higher on P5 and P8 

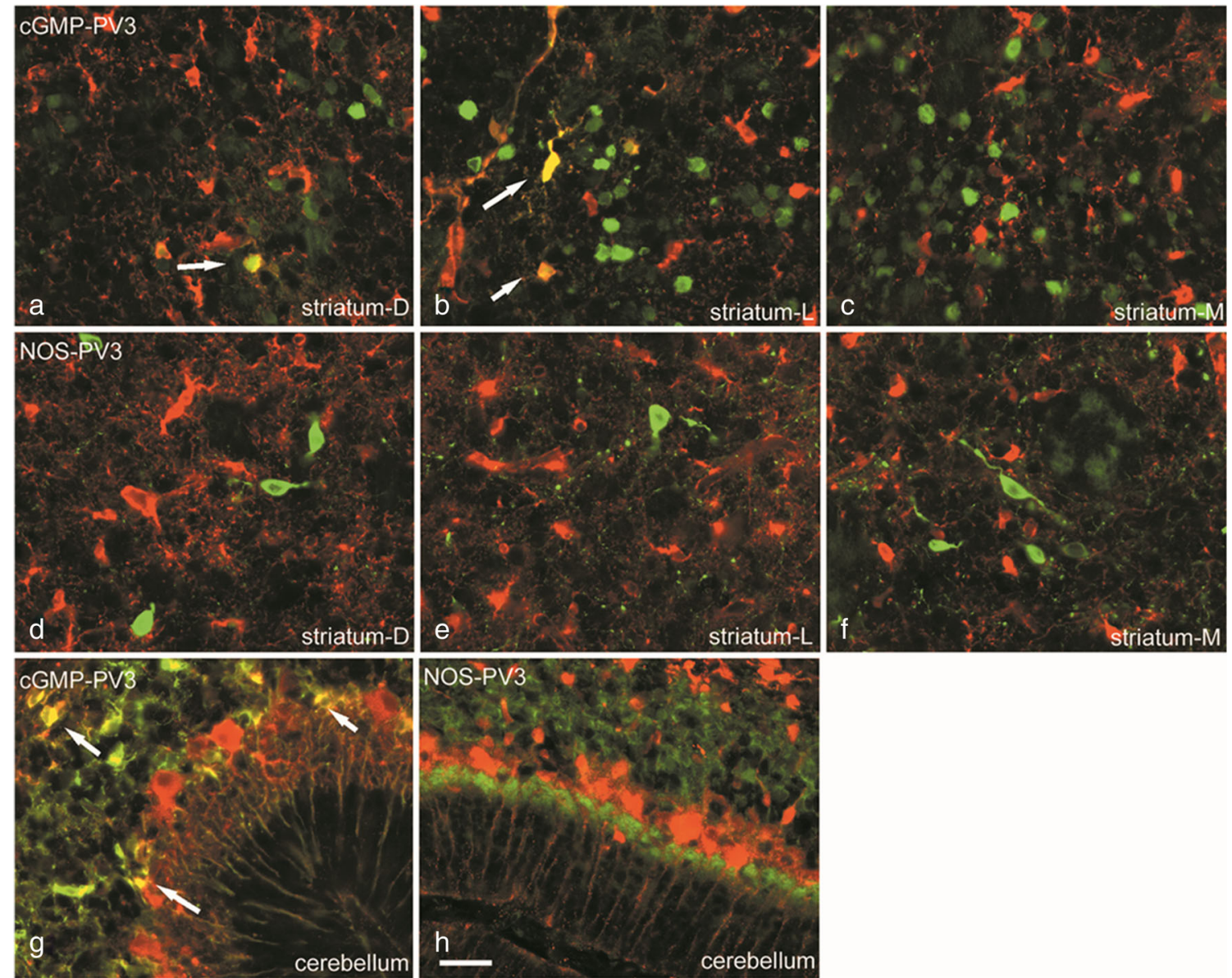

Fig. 3 Double labelling of parvalbumin (PV-3, in green) and NO production markers, cGMP or nNOS (in red). Yellow cells indicate double labelling and thus co-localization. Double labelling of PV-3 and

cGMP in the dorsal (a), lateral (b) and medial striatum (c) and cerebellum (g). Double labelling of PV-3 and nNOS in the dorsal (e), lateral (f) and medial (g) striatum and cerebellum (h). Scale bar is $50 \mu \mathrm{m}$

than on P12. The effect of PA on NO was thus maximal during first postnatal week. The NO/peroxynitrite production was

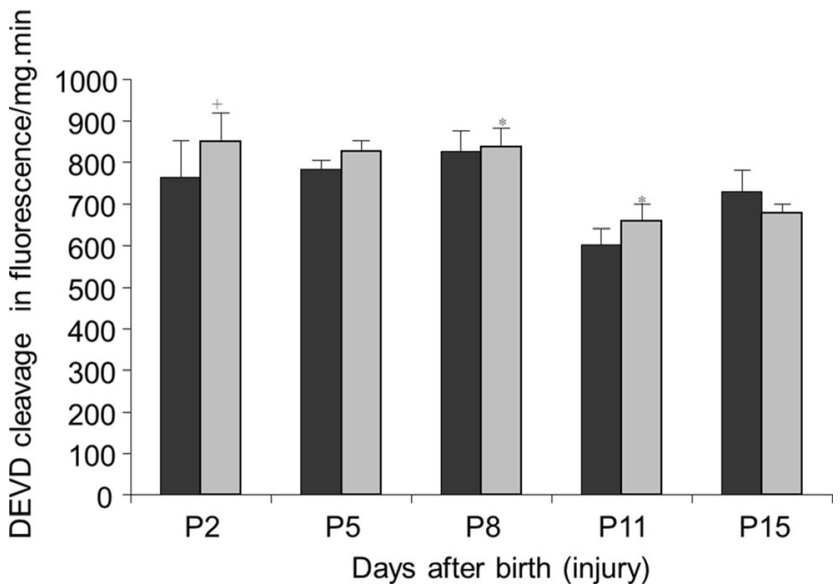

Fig. 4 Caspase-3-like activity (DEVD cleavage, expressed in cleaved $\mathrm{AMC}$ fluorescence per $\mathrm{mg}$ wet weight per minute) within the cerebellum of control (in black) and asphyctic (in grey) rats during the first 15 days after birth. The caspase-3-like activity after global asphyxia was compared using a two-way ANOVA with a Bonferroni correction for repeated measures. (Plus sign) $P=0.06$, (asterisk) $P<0.05$

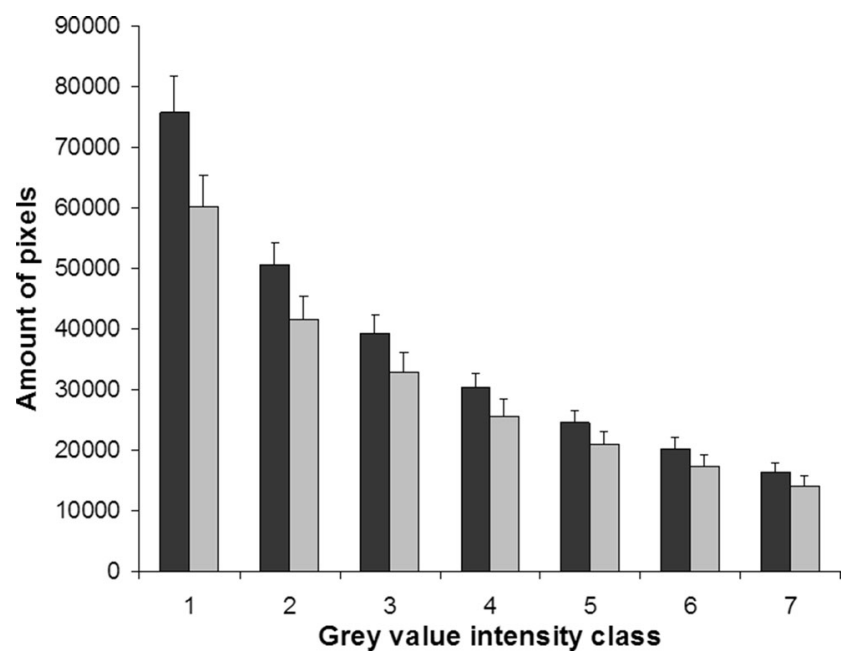

Fig. 5 Histogram of division of pixels over intensity classes of GFAP immunoreactivity in striata of control (shown in black, $n=5$ ) and asphyctic rats (shown in grey, $n=5$ ), at P12. Immunofluorescence intensity was converted to grey values divided over 16 classes. The results of classes 10-16 are shown as almost no pixels were present in other classes. There was no difference in distribution or amount of pixels per grey value intensity class between the groups. Data are expressed as mean \pm SEM 
higher in the cerebellum than that in the striatum at all timepoints, but PA did not cause a supraphysiologic increase on top of the NO production seen in the control groups.

In both the striatum and cerebellum, there are subpopulations of neurons that would be vulnerable to supraphysiologic NO production - and relatively resistant neurons that express nNOS. Ninety-five percent of the striatal neurons are GABAergic projection neurons (medium spiny neurons), and the remaining $5 \%$ is made up by interneurons. The interneurons are divided into tonically active cholinergic neurons, fast-spiking GABAergic interneurons, which express parvalbumin, and low-threshold spiking interneurons, which express somatostatin, neuropeptide $\mathrm{Y}$ and nNOS. The lowthreshold spiking interneurons are the primary source of striatal nNOS (Lenz and Lobo 2013; Liljeholm and O'Doherty 2012). nNOS is maximally expressed in the striatal regions where immature NMDA receptors are expressed (Black et al. 1995). Upon excessive stimulation of the NMDA receptors during PA, peroxynitrite is increased in the region (Ferriero et al. 1990; McQuillen and Ferriero 2004). The NO-producing interneurons are resistant to PA and NMDA-mediated excitotoxicity. However, the nearby striatal projection neurons are vulnerable to increased peroxynitrite formation. This selective vulnerability of the striatal projection neurons may result from a bystander effect attributable to their proximity to the enriched population of nNOS-expressing interneurons neurons (Ehrlich 2012; McQuillen and Ferriero 2004; Titomanlio et al. 2015). The relative resistance of the low-threshold spiking nNOS interneurons is echoed by our observations. The nNOS immunoreactivity did not change, and thus presumably PA did not alter the amount of nNOSexpressing interneurons in the striatum. However, $\mathrm{NO} /$ peroxynitrite and cGMP increased in the striatum and cGMP partially co-localized with parvalbumin neurons (Fig. 3), which indicates that there was more $\mathrm{NO}$ available to the parvalbuminergic neurons. Parvalbumin fast-spiking and cholinergic interneurons in the striatum have demonstrated vulnerability to PA (de Vente et al. 2000; Kohlhauser et al. 1999; Van de Berg et al. 2002).

In the cerebellum, nNOS is expressed by both the mature granule neurons and the molecular layer interneurons (basket and stellar cells) (Contestabile 2012) and these cells are thus relatively more resistant to $\mathrm{NO} /$ peroxynitrite toxicity. The Purkinje cell population (which express parvalbumin, but not nNOS) is sensitive to peroxynitrite, but these cells also need a small amount of NO to survive (Oldreive et al. 2012). During normal postnatal development, the expression and activity of cerebellar nNOS increases slowly throughout the first week. The granule neurons only show detectable NOS reactivity after migrating to the internal granular layer (Contestabile 2012). We observed $\mathrm{NO} /$ peroxynitrite production throughout the molecular and granular layer of the cerebellum in both control and PA rats. nNOS expression and cGMP production occurred throughout the cerebellum in both groups at $35.5^{\circ} \mathrm{C}$. Lowering the temperature by $10^{\circ} \mathrm{C}$ drastically lowered NO production. This correlates with the clinical observations that selective head cooling is beneficial after PA (Guillet et al. 2012).

We only observed changes in the cerebellar caspase-3-like activity in the second week of postnatal life in both control and asphyctic rats. The pattern of caspase-3-like activity in the cerebellum is unique in that it declines after the first week of postnatal life, but increases again afterwards (P15). There was a modest increase in caspase- 3 activity in the asphyctic groups up until P11, but overall, the patterns of caspase-3-like activity (and distribution of caspase-3 immunoreactive cells) was similar between groups and probably reflects physiological cell death. Apoptotic cell death is crucial for the normal development of the CNS and occurs in all brain areas during foetal and postnatal life (Devoto et al. 2013).

Although the striatum as a whole is susceptible to PA, not all striatal neurons are affected equally after PA. The nNOSexpressing interneurons were spared in our model. This is also seen in chronic degenerative diseases of the striatum that lead difficulties in motor control, like Huntington's disease. In Huntington's, the striatal neurons that do not release NO degenerate during the disease course, but the nNOS-producing interneurons are spared. The striatal projection neurons that form cortico-striatal network are particularly sensitive to glutamate-induced excitotoxicity and NO release (Canzoniero et al. 2014; Ehrlich 2012).

Previous NO-related research in the same model of asphyxia showed that increased striatal cGMP production is still evident at P10 (Loidl et al. 1998). However, directly after PA, when the foetal circulation and metabolism is still maintained, there were no apparent changes in the activity or transcription of nNOS (Lubec et al. 1999) or inducible NOS (Calamandrei et al. 2004). Early intervention in NO production could have long-term therapeutic effects. At 6 months, PA-related increases in striatal NOS activity were still apparent. The NOS-containing neurons had ultra-structural changes including cytomegaly, and the surrounding neurons showed increased degeneration (Capani et al. 1997). In the same regions, neurons that express nicotinamide adenine dinucleotide phosphate diaphorase (NADPH-d) - a marker of NO synthesishad similar cytomegalic morphology. The gross amount of NADPH-d expressing neurons was not altered by PA (Loidl et al. 1997). The results of the timing data suggest that the optimal therapeutic window for a NO-targeted therapy in the preterm infant would be in the first week after PA. Overall, our results show that reducing $\mathrm{NO}$ production could be a potential therapy to reduce striatal damage. The protective effects of NO-reduction may be limited to the regions with supraphysiological increases in NO. 


\section{Conclusion}

From these observations, we conclude that PA has a greater impact on $\mathrm{NO} /$ peroxynitrite production in the striatum than that in the cerebellum. Although NO/peroxynitrite was observed throughout the cerebellum, this can be ascribed to the high nNOS activity of the region. The greatest increase in NO production occurred during the first week, although it was previously reported that this change is not evident directly after PA. A therapeutic window exists within the first week after PA, during which inhibitors of NO production would likely have their best therapeutic effect in the preterm brain. Although this type of intervention would likely only show regional benefits in the brain regions with lower initial NO production, attenuating striatal neuron loss could have a motor-benefit after PA. Astrocytes were not notably increased by $\mathrm{PA}$, thus the striatal $\mathrm{NO}$ /peroxynitrite increase is likely independent of infiltration by inflammatory cells. Targeting inflammation alone is likely to be ineffective after PA, and alternate pathways should be considered.

Acknowledgements This research was partially supported by the Sistema de Investigación y Desarrollo (SINDE) of the Universidad Católica de Santiago de Guayaquil, Guayaquil, Ecuador, through the grant No SIU- 319: Perinatal asphyxia and stem cell treatment and the Education, Audiovisual and Culture Executive Agency, EU through the grant No 2013-3396. The generous gift of the antiserum against parvalbumin by Dr. Piers Emson is gratefully acknowledged. The authors are grateful to L. Veerbeek, M. Huiban-Minnaar, M.O. Lopez-Figueroa, M. Markerink and H.P.J. Steinbusch for their expert technical assistance and helpful suggestions. M. Barkhuizen is funded by the National Research Foundation of South Africa (Grant specific reference numbers 89230 and 98217). All views expressed in this article are those of the authors and not of the funding agencies.

\section{Compliance with Ethical Standards}

Ethical Approval All applicable international, national and/or institutional guidelines for the care and use of animals were followed. All procedures performed in the studies involving animals were in accordance with the ethical standards of Maastricht University at which the studies were conducted.

Open Access This article is distributed under the terms of the Creative Commons Attribution 4.0 International License (http:// creativecommons.org/licenses/by/4.0/), which permits unrestricted use, distribution, and reproduction in any medium, provided you give appropriate credit to the original author(s) and the source, provide a link to the Creative Commons license, and indicate if changes were made.

\section{References}

Armstrong-Wells J, Bernard TJ, Boada R, Manco-Johnson M (2010) Neurocognitive outcomes following neonatal encephalopathy. NeuroRehabilitation 26:27-33

Balleine BW, Delgado MR, Hikosaka O (2007) The role of the dorsal striatum in reward and decision-making. J Neurosci 27:8161-8165
Black SM, Bedolli MA, Martinez S, Bristow JD, Ferriero DM, Soifer SJ (1995) Expression of neuronal nitric oxide synthase corresponds to regions of selective vulnerability to hypoxia-ischaemia in the developing rat brain. Neurobiol Dis 2:145-155

Blanco S et al (2010) Study of the nitric oxide system in the rat cerebellum during aging. BMC Neurosci 11:78

Bryan NS, Grisham MB (2007) Methods to detect nitric oxide and its metabolites in biological samples. Free Radic Biol Med 43:645-657

Cabaj A, Bekiesińska-Figatowska M, Mądzik J (2012) MRI patterns of hypoxic-ischemic brain injury in preterm and full term infantsclassical and less common MR findings. Pol J Radiol 77:71-76

Calamandrei G, Venerosi AP, Valanzano A, De Berardinis MA, Greco A, Puopolo M, Minghetti L (2004) Increased brain levels of F2isoprostane are an early marker of behavioral sequels in a rat model of global perinatal asphyxia. Pediatr Res 55:85-92

Canzoniero LM, Granzotto A, Turetsky DM, Choi DW, Dugan LL, Sensi SL (2014) nNOS (+) striatal neurons, a subpopulation spared in Huntington's Disease, possess functional NMDA receptors but fail to generate mitochondrial ROS in response to an excitotoxic challenge Mitochondria: the cell powerhouse and nexus of stress. 64

Capani F, Loidl F, Lopez-Costa JJ, Selvin-Testa A, Saavedra JP (1997) Ultrastructural changes in nitric oxide synthase immunoreactivity in the brain of rats subjected to perinatal asphyxia: neuroprotective effects of cold treatment. Brain Res 775:11-23

Charriaut-Marlangue $C$ et al (2012) Inhaled nitric oxide reduces brain damage by collateral recruitment in a neonatal stroke model. Stroke 43:3078-3084

Contestabile A (2012) Role of nitric oxide in cerebellar development and function: focus on granule neurons. Cerebellum 11:50-61

De Smet HJ, Paquier P, Verhoeven J, Mariën P (2013) The cerebellum: its role in language and related cognitive and affective functions. Brain Lang 127:334-342

de Vente J, Steinbusch HW, Schipper J (1987) A new approach to immunocytochemistry of 3',5'-cyclic guanosine monophosphate: preparation, specificity, and initial application of a new antiserum against formaldehyde-fixed 3',5'-cyclic guanosine monophosphate. Neuroscience 22:361-373

de Vente J, Markerink-van Ittersum M, van Abeelen J, Emson PC, Axer H, Steinbusch HW (2000) NO-mediated cGMP synthesis in cholinergic neurons in the rat forebrain: effects of lesioning dopaminergic or serotonergic pathways on nNOS and cGMP synthesis. Eur J Neurosci 12:507-519

Devoto VP, Bogetti M, de Plazas SF (2013) Developmental and hypoxiainduced cell death share common ultrastructural and biochemical apoptotic features in the central nervous system. Neuroscience 252:190-200

Dorfman VB, Rey-Funes M, Bayona JC, López EM, Coirini H, Loidl CF (2009) Nitric oxide system alteration at spinal cord as a result of perinatal asphyxia is involved in behavioral disabilities: hypothermia as preventive treatment. J Neurosci Res 87:1260-1269

Drury PP et al (2014) nNOS inhibition during profound asphyxia reduces seizure burden and improves survival of striatal phenotypic neurons in preterm fetal sheep. Neuropharmacology 83:62-70

Edwards AD et al (2010) Neurological outcomes at 18 months of age after moderate hypothermia for perinatal hypoxic ischaemic encephalopathy: synthesis and meta-analysis of trial data. BMJ 340:c363. doi:10.1136/bmj.c363

Ehrlich ME (2012) Huntington's disease and the striatal medium spiny neuron: cell-autonomous and non-cell-autonomous mechanisms of disease. Neurotherapeutics 9:270-284

El-Khodor BF, Boksa P (2003) Differential vulnerability of male versus female rats to long-term effects of birth insult on brain catecholamine levels. Exp Neurol 182:208-219

Ergenekon E, Gücüyener K, Erbaș D, Aral S, Koç E, Atalay Y (2004) Cerebrospinal fluid and serum vascular endothelial growth factor and nitric oxide levels in newborns with hypoxic ischemic encephalopathy. Brain Dev 26:283-286 
Ferriero D, Arcavi L, Simon R (1990) Ontogeny of excitotoxic injury to nicotinamide adenine dinucleotide phosphate diaphorase reactive neurons in the neonatal rat striatum. Neuroscience 36:417-424

Freeman TB, Sanberg PR, Isacson O (1995) Development of the human striatum: implications for fetal striatal transplantation in the treatment of Huntington's disease. Cell Transplant 4:539-545

Groenendaal F, Lammers H, Smit D, Nikkels PG (2006) Nitrotyrosine in brain tissue of neonates after perinatal asphyxia. Archives of Disease in Childhood-Fetal and Neonatal Edition 91:F429-F433

Guillet R et al (2012) Seven- to eight-year follow-up of the CoolCap trial of head cooling for neonatal encephalopathy. Pediatr Res 71:205209. doi:10.1038/pr.2011.30

Gunes T, Ozturk MA, Koklu E, Kose K, Gunes I (2007) Effect of allopurinol supplementation on nitric oxide levels in asphyxiated newborns. Pediatr Neurol 36:17-24

Herbison AE, Simonian SX, Norris PJ, Emson PC (1996) Relationship of neuronal nitric oxide synthase immunoreactivity to GnRH neurons in the ovariectomized and intact female rat. J Neuroendocrinol 8:73-82

Ikeno S, Nagata N, Yoshida S, Takahashi H, Kigawa J, Terakawa N (2000) Immature brain injury via peroxynitrite production induced by inducible nitric oxide synthase after hypoxia-ischemia in rats. J Obstet Gynaecol Res 26:227-234

Ji H et al (2009) Selective neuronal nitric oxide synthase inhibitors and the prevention of cerebral palsy. Ann Neurol 65:209-217

Klawitter V, Morales P, Bustamante D, Goiny M, Herrera-Marschitz M (2006) Plasticity of the central nervous system (CNS) following perinatal asphyxia: does nicotinamide provide neuroprotection? Amino Acids 31:377-384

Klawitter V, Morales P, Bustamante D, Gomez-Urquijo S, Hökfelt T, Herrera-Marschitz M (2007) Plasticity of basal ganglia neurocircuitries following perinatal asphyxia: effect of nicotinamide. Exp Brain Res 180:139-152

Kohlhauser C, Mosgoeller W, Hoeger H, Lubec G, Lubec B (1999) Cholinergic, monoaminergic and glutamatergic changes following perinatal asphyxia in the rat. Cell Mol Life Sci 55:1491-1501

Lee AC et al (2013) Intrapartum-related neonatal encephalopathy incidence and impairment at regional and global levels for 2010 with trends from 1990. Pediatr Res 74:50-72

Lenz JD, Lobo MK (2013) Optogenetic insights into striatal function and behavior. Behav Brain Res 255:44-54

Liljeholm M, O'Doherty JP (2012) Contributions of the striatum to learning, motivation, and performance: an associative account. Trends Cogn Sci 16:467-475

Logitharajah P, Rutherford MA, Cowan FM (2009) Hypoxic-ischemic encephalopathy in preterm infants: antecedent factors, brain imaging, and outcome. Pediatr Res 66:222-229

Loidl CF, Capani F, López-Costa J, Selvín-Testa A, López EM, PecciSaavedra J (1997) Long term changes in NADPH-diaphorase reactivity in striatal and cortical neurons following experimental perinatal asphyxia: neuroprotective effects of hypothermia. Int J Neurosci 89:1-14

Loidl CF, De Vente J, van Ittersum MM, van Dijk EH, Vles JS, Steinbusch HW, Blanco CE (1998) Hypothermia during or after severe perinatal asphyxia prevents increase in cyclic GMP-related nitric oxide levels in the newborn rat striatum. Brain Res 791:303-307

Lopez-Figueroa MO, Caamano C, Morano MI, Ronn LC, Akil H, Watson SJ (2000) Direct evidence of nitric oxide presence within mitochondria. Biochem Biophys Res Commun 272:129-133

Lubec B et al (1999) Nitric oxide and nitric oxide synthase in the early phase of perinatal asphyxia of the rat. Neuroscience 93:1017-1023

McQuillen PS, Ferriero DM (2004) Selective vulnerability in the developing central nervous system. Pediatr Neurol 30:227-235

Oldreive CE, Gaynor S, Doherty GH (2012) Effects of nitric oxide on the survival and neuritogenesis of cerebellar Purkinje neurons. J Mol Neurosci 46:336-342

Perlman JM (2006) Intervention strategies for neonatal hypoxic-ischemic cerebral injury. Clin Ther 28:1353-1365
Piña-Crespo JC, Sanz-Blasco S, Lipton SA (2014) Concept of excitotoxicity via glutamate receptors. In: Handbook of neurotoxicity. Springer, pp 1015-1038

Rancillac A et al (2006) Glutamatergic control of microvascular tone by distinct GABA neurons in the cerebellum. J Neurosci 26:6997-7006

Rao $S$ et al (2011) Involvement of neuronal nitric oxide synthase in ongoing fetal brain injury following near-term rabbit hypoxia-ischemia. Dev Neurosci 33:288-298

Rodriguez J, Specian V, Maloney R, Jourd'heuil D, Feelisch M (2005) Performance of diamino fluorophores for the localization of sources and targets of nitric oxide. Free Radic Biol Med 38:356-368

Roychowdhury S, Luthe A, Keilhoff G, Wolf G, Horn TF (2002) Oxidative stress in glial cultures: detection by DAF-2 fluorescence used as a tool to measure peroxynitrite rather than nitric oxide. Glia 38:103-114

Sarnat HB, Auer RN, Flores-Sarnat L (2013) Synaptogenesis in the fetal corpus striatum, Globus pallidus, and substantia nigra correlations with Striosomes of Graybiel and Dyskinesias in premature infants. J Child Neurol 28:60-69

Schaaf J, Mol B, Abu-Hanna A, Ravelli A (2011) Trends in preterm birth: singleton and multiple pregnancies in the Netherlands, 2000-2007. BJOG: An International Journal of Obstetrics \& Gynaecology 118: 1196-1204

Schwaller B, Meyer M, Schiffmann S (2002) 'New'functions for 'old'proteins: the role of the calcium-binding proteins calbindin D-28 k, calretinin and parvalbumin, in cerebellar physiology. Studies with knockout mice. Cerebellum 1:241-258

Semple BD, Blomgren K, Gimlin K, Ferriero DM, Noble-Haeusslein LJ (2013) Brain development in rodents and humans: identifying benchmarks of maturation and vulnerability to injury across species. Prog Neurobiol 106:1-16

Shah DK et al (2006) Reduction in cerebellar volumes in preterm infants: relationship to white matter injury and neurodevelopment at two years of age. Pediatr Res 60:97-102

Shim S-Y, Kim H-S (2013) Oxidative stress and the antioxidant enzyme system in the developing brain. Korean journal of pediatrics 56:107-111

Titomanlio L, Fernández-López D, Manganozzi L, Moretti R, Vexler ZS, Gressens P (2015) Pathophysiology and neuroprotection of global and focal perinatal brain injury: lessons from animal models. Pediatr Neurol 52:566-584

Tzvi E, Münte TF, Krämer UM (2014) Delineating the cortico-striatalcerebellar network in implicit motor sequence learning. NeuroImage 94:222-230

Van de Berg WD, Schmitz C, Steinbusch HW, Blanco CE (2002) Perinatal asphyxia induced neuronal loss by apoptosis in the neonatal rat striatum: a combined TUNEL and stereological study. Exp Neurol 174:29-36

Van den Hove D et al (2006) Prenatal stress and neonatal rat brain development. Neuroscience 137:145-155

Vlassaks E, Brudek T, Pakkenberg B, Gavilanes AW (2014) Cerebellar cytokine expression in a rat model for fetal asphyctic preconditioning and perinatal asphyxia. Cerebellum 13:471-478

Volpe JJ (2009a) Brain injury in premature infants: a complex amalgam of destructive and developmental disturbances. The Lancet Neurology 8:110-124

Volpe JJ (2009b) Cerebellum of the premature infant: rapidly developing, vulnerable, clinically important. J Child Neurol 24:1085-1104

Volpe JJ (2012) The neurological outcome of perinatal asphyxia early brain damage V1: research orientations and clinical observations. 151

Weis SN et al (2011) Early biochemical effects after unilateral hypoxiaischemia in the immature rat brain. Int J Dev Neurosci 29:115-120

Yu L, Derrick M, Ji H, Silverman RB, Whitsett J, Vásquez-Vivar J, Tan S (2011) Neuronal nitric oxide synthase inhibition prevents cerebral palsy following hypoxia-ischemia in fetal rabbits: comparison between JI-8 and 7-nitroindazole. Dev Neurosci 33:312-319

Zhu C, Xu F, Wang X, Shibata M, Uchiyama Y, Blomgren K, Hagberg H (2006) Different apoptotic mechanisms are activated in male and female brains after neonatal hypoxia-ischaemia. J Neurochem 96:1016-1027 